Authors have nothing to disclose with regard to commercial support.

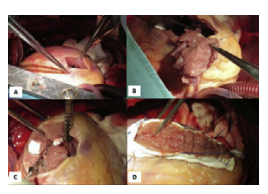

\section{ELECTRICAL STORMS \\ IN PATIENTS WITH HYPERTROPHIC CARDIOMYOPATHY: DO WE HAVE A SOLUTION? \\ To the Editor:}

We read the case series on electrical storms in patients with hypertrophic cardiomyopathy (HCM) with midventricular obstruction and apical aneurysm.

The prevalence of arrhythmias is seen throughout the spectrum of HCM phenotype, the most catastrophic event being sudden cardiac death (SCD). Arrhythmias include atrial fibrillation, ventricular ectopics, nonsustained ventricular tachycardia, and malignant ventricular tachyarrhythmias. The indication for an implantable cardioverterdefibrillator (ICD) is clear when a patient presents with a history of syncope and the Holter monitoring demonstrates nonsustained ventricular tachycardia. There have been no randomized studies or validated prospective risk prediction models to guide ICD implantation for primary prevention in $\mathrm{HCM}$. The binary risk assessment approaches traditionally used in patients with HCM to decide the appropriateness of primary prevention ICD implantation are inconsistent and do not take into account different effect size of individual risk factors, providing only crude estimates of risk. The search for the risk prediction model has evaluated several factors. None of the 12-lead surface electrocardiographic markers were associated with SCD (markedly increased voltages, QRS duration, left or rightward QRS axis, abnormal Q waves, and QTc or QT dispersion). ${ }^{2}$ Although rare, the presence exercise-induced nonsustained VT or ventricular fibrillation was associated with a 3.73 -fold increase in the risk of SCD. ${ }^{3}$ Delayed enhancement on cardiac magnetic resonance is strongly associated with fibrosis and myocyte disarray, which correlated with nonsustained VT on Holter and predicted risk of SCD. ${ }^{4}$ Even in asymptomatic or mildly symptomatic patients with HCM, this finding

\footnotetext{
The Editor welcomes submissions for possible publication in the Letters to the Editor section that consist of commentary on an article published in the Journal or other relevant issues. Authors should: • Include no more than 500 words of text, three authors, and five references. • Type with double-spacing. • See http://jtcs.ctsnetjournals.org/ misc/ifora.shtml for detailed submission instructions. • Submit the letter electronically via jtcvs.editorialmanager.com. Letters commenting on an article published in the JTCVS will be considered if they are received within 6 weeks of the time the article was published. Authors of the article being commented on will be given an opportunity of offer a timely response ( 2 weeks) to the letter. Authors of letters will be notified that the letter has been received. Unpublished letters cannot be returned.
}

was associated with a significantly increased frequency of ventricular tachyarrhythmias on Holter monitoring compared with those without it. The complex phenotype of HCM has several genetic and nongenetic factors responsible, which precludes the use of a predictive risk assessment model for SCD based on genotype alone.

In 2014, O'Mahony and colleagues ${ }^{5}$ proposed the first validated prognostic risk prediction model for SCD in HCM (called "HCM risk-SCD") based on data from a retrospective, multicenter longitudinal cohort study. The investigators predict that if 16 patients with $\mathrm{HCM}$ receive an ICD as a result of a 5-year SCD risk estimate of $4 \%$ or more using their prediction tool, 1 death will be prevented at 5 years. One of the confounding factors of this study was that by using this model, the calculated risk of SCD decreased in patients with severe left ventricular hypertrophy $(>35 \mathrm{~mm})$. The decrease in risk in patients with extreme left ventricular hypertrophy may have been due to the small number of patients in this category. This tool cannot be used in the pediatric age group, in syndromic patients with Noonan's syndrome, and in patients who have undergone myectomy or alcohol septal ablation.

In this case series, resection of the apical aneurysm and midventricular myectomy was associated with cessation of the arrhythmias. There was extensive scar tissue in the aneurysm validating that the arrhythmic substrate in these patients is linked to scar and myocyte disarray within the hypertrophied areas. The rare instances of VT after myectomy or alcohol septal ablation requiring ICD implantation may be linked to procedure related scar. Therefore, a risk prediction model for SCD in HCM may need to factor scar as a major arrhythmic substrate.

$$
\begin{array}{r}
\text { Srilakshmi M. Adhyapak, DNB }{ }^{a} \\
\text { V. Rao Parachuri, FRCS, CTh }{ }^{b} \\
{ }^{a} \text { Department of Cardiology } \\
\text { St John's Medical College Hospital } \\
\text { Bangalore, India } \\
{ }^{b} \text { Department of Cardiothoracic Surgery } \\
\text { Narayana Hrudayalaya Institute of Medical Sciences } \\
\text { Bangalore, India }
\end{array}
$$

\section{References}

1. Nguyen A, Schaff HV. Electrical storms in patients with apical aneurysms and hypertrophic cardiomyopathy with midventricular obstruction: a case series. J Thorac Cardiovasc Surg. 2017;154:101-3.

2. Sherrid MV, Cotiga D, Hart D, Ehlert F, Haas TS, Shen WK, et al. Relation of 12 lead electrocardiogram patterns to implanted defibrillator-terminated ventricular tachyarrhythmias in hypertrophic cardiomyopathy. Am J Cardiol. 2009;104:1722.

3. Gimeno JR, Tomé-Esteban M, Lofiego C, Hurtado J, Pantazis A, Mist B, et al. Exercise-induced ventricular arrhythmias and risk of sudden cardiac death in patients with hypertrophic cardiomyopathy. Eur Heart J. 2009;30:2599-605.

4. Adabag AS, Maron BJ, Appelbaum E, Harrigan CJ, Buros JL, Gibson CM, et al. Occurrence and frequency of arrhythmias in hypertrophic cardiomyopathy in relation to delayed enhancement on cardiovascular magnetic resonance. J Am Coll Cardiol. 2008;51:1369-74. 
5. O’Mahony C, Jichi F, Pavlou M, Monserrat L, Anastasakis A, Rapezzi C, et al. A novel clinical risk prediction model for sudden cardiac death in hypertrophic cardiomyopathy (HCM risk-SCD). Eur Heart J. 2014;35:2010-20.

https://doi.org/10.1016/j.jtcvs.2017.12.040

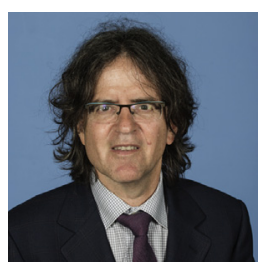

A DEDICATED HEART TEAM IS NEEDED TO MAXIMIZE THE TREATMENT OF PATIENTS WITH HYPERTROPHIC CARDIOMYOPATHY

Reply to the Editor:

The letter by Dr Srilakshmi is a thoughtful representation of the current challenges we are all facing in treating patients with hypertrophic cardiomyopathy (HCM). These patients, when diagnosed or become symptomatic, pose a unique challenge in achieving better control of symptoms and minimizing the risk of sudden death. The issue raised by Dr Srilakshmi of risk prediction to better guide the use of an automatic implantable cardioverter-defibrillator is an interesting one. However, it remains theoretical only because no risk prediction model is perfect, let alone one with such a small number of patients available. I agree that the indications to use automatic implantable cardioverter-defibrillators to minimize the risk of sudden death due to malignant ventricular arrhythmia may not always be clear, but the risk-benefit considerations are well accepted among the medical community and the patients. In the case series published by Drs Nguyen and Schaff from the Mayo Clinic, it was clearly demonstrated that surgical treatment can be safe and effective in many cases and especially for those with midventricular obstruction and malignant arrhythmias.

Currently, the approach to HCM in most centers does not involve a true heart team with a thorough discussion and planning of each case. It is clear that there needs to be improved communication and refinement in the clinical approach to such patients. One of the challenges that most centers face is the small number of patients treated and the lack of significant clinical experience, especially when it comes to midventricular lesions. In general, the surgical community is not familiar with the surgical approaches appropriate for this subgroup of patients, mainly because of the rarity of HCM. As a subspecialty, it is imperative to recognize that a well-skilled surgical team can perform high-risk procedures with outstanding outcomes. Therefore, there is a need to approach HCM as a team to better understand the genetics, the heterogeneous clinical presentation, and all the different treatment options, including surgery. That being said, a good and reliable risk algorithm is
Author has nothing to disclose with regard to commercial support.

always necessary and should be incorporated in our clinical practice if proven to improve patient outcomes.

Niv Ad, MD

Department of Cardiovascular and Thoracic Surgery

West Virginia University

Morgantown, WVa

https://doi.org/10.1016/j.jtcvs.2018.02.075

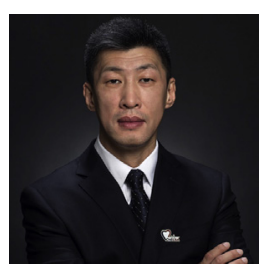

\section{STRAIGHT DEEP \\ HYPOTHERMIC CIRCULATORY \\ ARREST: CLING TO OLD FASHION OR NOT?}

To the Editor:

We read with great interest the study by Damberg and colleagues ${ }^{1}$ showing excellent results after aortic surgery with straight deep hypothermic circulatory arrest (DHCA) without cerebral perfusion. Antegrade cerebral perfusion (ACP) was not performed to avoid potential air/particulate embolism and hypoperfusion or hyperperfusion. They demonstrated that straight DHCA (DHCA time within 50 minutes) was a safe and effective method of brain protection in patients at their center, associated with favorable short-term mortality (2.9\%) and stroke rate $(2 \%)$, as well as late survival (estimated 1and 5-year survivals $92.2 \%$ and $81.5 \%$, respectively), which was comparable to outcomes in other cohorts using ACP.

However, we suppose these favorable results could be achieved only at the best hands among selected patients. The generality of their conclusion should be questioned. Under some circumstances, such as patients aged more than 60 years, redo surgery, emergency surgery, dissection, total arch replacement, and junior surgeon at the deep portion of the learning curve, it is reasonable to provide an extra layer of brain protection by ACP.

To the best of our knowledge, adjunctive ACP has been shown to be superior over straight DHCA by single-center cohort studies: a large-scale comparative effectiveness study from the Society of Thoracic Surgeons database ${ }^{2}$ and a meta-analysis. ${ }^{3}$ During circulatory arrest, a sharp decrease of cerebral oxygenation was observed in DHCA compared with ACP, increasing the risk of neurologic complications. ${ }^{4}$ Furthermore, deep 